\title{
Managing Obesity and Related Comorbidities: A Potential Pharmacological Target in the Adenosine System?
}

\author{
Vanessa D'Antongiovanni ${ }^{1}$, Matteo Fornai ${ }^{1}$, Carolina Pellegrini ${ }^{2}$, Corrado Blandizzi ${ }^{1 *}$ and \\ Luca Antonioli ${ }^{1}$
}

${ }^{1}$ Department of Clinical and Experimental Medicine, University of Pisa, Pisa, Italy, ${ }^{2}$ Department of Pharmacy, University of Pisa, Pisa, Italy

Keywords: adenosine, adenosine receptors, obesity, adipogenesis and lipogenesis, diabete mellitus, cardiovascular diseases, gastrointestinal dysfunction, cognitive impairment

\section{OPEN ACCESS}

Edited by:

Francesco Caciagli,

University of Studies G. d'Annunzio

Chieti and Pescara, Italy

Reviewed by:

Rajaraman D. Eri,

University of Tasmania, Australia Valeria Marzano,

Bambino Gesù Children Hospital (IRCCS), Italy

*Correspondence:

Corrado Blandizz

c.blandizzi@gmail.com

Specialty section: This article was submitted to Experimental Pharmacology and

Drug Discovery,

a section of the journal

Frontiers in Pharmacology

Received: 27 October 2020 Accepted: 21 December 2020

Published: 18 January 2021

Citation:

D'Antongiovanni V, Fornai M, Pellegrini C, Blandizzi $C$ and Antonioli L (2021) Managing Obesity and Related

Comorbidities: A Potential

Pharmacological Target in the

Adenosine System?.

Front. Pharmacol. 11:621955.

doi: 10.3389/fphar.2020.621955

\section{INTRODUCTION}

Obesity is a complex and multifactorial disease characterized by abnormal fat accumulation resulting from a disequilibrium between energy intake and its consumption (Bluher, 2019). At present, more than one-third of the world population is classified as obese or overweight, and according to a recent estimation it is expected that by 2030 this value will surpass 50\% (Bluher, 2019).

Obesity is associated with a condition of low-grade systemic inflammation that seems to be a common root to the onset and progression of several comorbidities, such as type-2 diabetes mellitus (T2DM), cardiovascular diseases (CVDs) and cognitive impairment (Jarolimova et al., 2013). In parallel, there is evidence that obese patients often complain of chronic gastrointestinal (GI) disturbances, including gastroesophageal reflux, diarrhea and constipation, undermining their quality of life (Camilleri et al., 2017; D'Antongiovanni et al., 2020a).

Preclinical studies on mice fed with high-fat diet (HFD) have shown that the adipocytes and adipose tissue-associated macrophages release a plethora of inflammatory mediators, including interleukin (IL)-1, IL-6, tumor necrosis factor (TNF) and monocyte chemoattractant protein-1, leading to a condition of systemic inflammation and oxidative stress (Ellulu et al., 2017). The chronicization of this inflammatory condition then affects the homeostatic mechanisms, and thereby the physiological functions, of several organs, leading to the development of different obesityassociated comorbidities (Ellulu et al., 2017). At present, the available pharmacological tools to manage obesity are unsatisfactory in terms of efficacy, safety and long-term maintenance of weight loss. Therefore, the identification of novel molecular targets, aimed at developing innovative antiobesity treatments, represents a challenging and exciting field of high scientific interest.

Over the years, several lines of evidence have outlined a critical role of the adenosine system in glucose homeostasis, inflammation, adipogenesis, insulin resistance and thermogenesis, thus suggesting an involvement of adenosine in the onset and progression of obesity (Pardo et al., 2017; D'Antongiovanni et al., 2020b). Indeed, the wide distribution of adenosine receptors (ARs) in tissues tightly involved in metabolism regulation leads to hypothesize the pharmacological modulation of adenosine pathways as a viable way to counteract obesity and related comorbidities (Hasko et al., 2008; Antonioli et al., 2011; Kotanska et al., 2020). In line with this view, encouraging preclinical data have reported beneficial effects of treatments with specific AR ligands in stemming adipose tissue inflammation and insulin resistance (Figler et al., 2011; Csoka et al., 2014). 
Based on the above background, the present opinion paper has been conceived to provide a critical appraisal of the available knowledge about the involvement of adenosine system in the pathophysiological mechanisms underlying obesity, pointing out its potential as therapeutic target to develop innovative therapeutic strategies aimed at counteracting the obesityassociated diseases.

\section{ADENOSINE SYSTEM: ENZYMES, TRANSPORTERS, RECEPTORS AND PHYSIOLOGICAL FUNCTIONS}

Adenosine is an endogenous nucleoside derived from the metabolism of adenosine triphosphate (ATP) (Sheth et al., 2014). Physiologically, adenosine is present at low levels in the interstitial fluids of unstressed tissues, with its concentration increasing quickly in the presence of metabolically stressful conditions (D’Antongiovanni et al., 2020b). Intracellular adenosine is produced from S-adenosylhomocysteine via S-adenosylhomocysteine hydrolase (Sheth et al., 2014). In the extracellular space, adenosine results mainly by the dephosphorylation of ATP, mediated in a sequential manner by ecto-nucleotide triphosphate diphosphohydrolase-1 (also named CD39) and by ecto- $5^{\prime}$-nucleotidase (also named CD73) (Eltzschig, 2013; Antonioli et al., 2013) (Figure 1A). The extracellular and intracellular adenosine levels are finely tuned by the activity of the nucleoside transporters classified into: equilibrative nucleoside transporters (ENTs), which transport nucleosides across cell membranes in either directions, and concentrative nucleoside transporters (CNTs), which shunt extracellular adenosine into the intracellular space against their concentration gradient (Pastor-Anglada et al., 2018). Another critical checkpoint in the regulation of adenosine levels is represented by the adenosine deaminase (ADA), a key enzyme involved in degradation of adenosine into inosine (Antonioli et al., 2012) (Figure 1A).

Most of the biological actions of adenosine are mediated by four different subtypes of $\mathrm{G}$-protein coupled receptors, that either inhibit $\left(\mathrm{A}_{1} \mathrm{R}\right.$ and $\left.\mathrm{A}_{3} \mathrm{R}\right)$ or stimulate $\left(\mathrm{A}_{2 \mathrm{~A}} \mathrm{R}\right.$ and $\left.\mathrm{A}_{2 \mathrm{~B}} \mathrm{R}\right)$ adenylyl cyclase activity and cAMP production in the cell. All ARs are coupled also to MAPK pathways, including $\mathrm{ERK}_{1 / 2}$ and p38 MAPK (Sheth et al., 2014) (Figure 1A). In addition, adenosine can also exerts receptorindependent effects, via less defined intracellular mechanisms, including the S-adenosylhomocysteine hydrolase systems, adenosine kinase and AMP-activated protein kinase (AMPK) (Da Silva et al., 2006; Boison, 2013). Once released into the extracellular space, adenosine takes a pivotal part in modulating a wide variety of physiological processes, including gut motility, blood flow, lipolysis and gluconeogenesis (Figure 1B).

\section{ROLE OF ADENOSINE IN ADIPOGENESIS AND LIPOLYSIS}

Adipogenesis is a physiological process of cell differentiation by which preadipocytes differentiate into adipocytes, with a consequent increase in the formation of white adipose tissue
(WAT) (Ouchi et al., 2011). An abnormal increment of WAT results in an increased release of pro-inflammatory cytokines along with altered adipokine secretion (Ouchi et al., 2011). In this context, adenosine, released continuously from adipocytes, is able to promote adipogenesis and lipid accumulation via $\mathrm{A}_{1} \mathrm{R}$ (Gharibi et al., 2012; Burnstock and Gentile, 2018). By contrast, the engagement of $\mathrm{A}_{2 \mathrm{~B}} \mathrm{Rs}$ is followed by the inhibition of preadipocyte differentiation, indicating a differential role of adenosine on adipogenesis, based on the receptor subtype engaged (Eisenstein et al., 2014).

It is worth to note that ARs play also important roles in controlling the functions of brown adipocytes. The brown adipose tissue (BAT), critically involved in thermogenesis and lipolysis, is emerging as a potential target for anti-obesity therapies (Cully, 2014). It is well acknowledged that $A_{1} R$ activation can blunt the lipolysis of brown adipocytes in rodents (Mohell, 1984). However, recent studies allowed to observe that $\mathrm{A}_{2 \mathrm{~A}} \mathrm{Rs}$, expressed mainly on brown adipocytes, are involved markedly in the process of thermogenesis (Cully, 2014; Gnad et al., 2014). In this regard, Gnad et al. observed that the pharmacological inhibition of $\mathrm{A}_{2 \mathrm{~A}} \mathrm{Rs}$, by its antagonist MSX-3, as well as the genetic deletion of these receptors in mice induced a decrease in BAT-dependent thermogenesis (Gnad et al., 2014). By contrast, treatment with $\mathrm{A}_{2 \mathrm{~A}} \mathrm{R}$ agonist, CGS21680, significantly increased energy expenditure, demonstrating the relevant contribution of $\mathrm{A}_{2 \mathrm{~A}} \mathrm{R}$ in mediating the thermogenic response (Gnad et al., 2014). In the same study, the authors also showed that the systemic administration of CGS21680, beyond to reduce the body weight gain, induced the browning of WAT and improved glucose tolerance in HFD mice (Gnad et al., 2014). Taken together, these results demonstrated that $\mathrm{A}_{2 \mathrm{~A}} \mathrm{R}$ activation triggers lipolysis, energy expenditure and browning of WAT, indicating a beneficial effect in counteracting the HFD-induced obesity in mice.

\section{OBESITY-RELATED COMORBIDITIES: ROLE OF ADENOSINE}

\section{Type-2 Diabetes Mellitus}

T2DM is a pathological condition, often associated with obesity, characterized by impairment of insulin secretion and/or function (Hardy et al., 2012). In obese patients, the underlying chronic low-grade inflammatory condition represents a pivotal factor in the onset of insulin resistance, a prodromal step leading to the development of T2DM (Nowotny et al., 2015). As a consequence of insulin resistance, a compensatory hyperinsulinemia occurs, with an over-stimulation of pancreatic $\beta$-cell function, followed by their exhaustion (Nowotny et al., 2015).

Several studies have reported a relationship between the adenosine system and obesity-induced insulin resistance and T2DM. Some authors observed that the over-expression of $A_{1} R s$ in the adipose tissue of knock-in mice protected from HFD-induced insulin resistance, indicating this receptor subtype as a potential therapeutic target for the management of obesity-related insulin resistance and T2DM (Dong et al., 2001). However, it is noteworthy the $A_{2 B} R$ subtype seems to play 


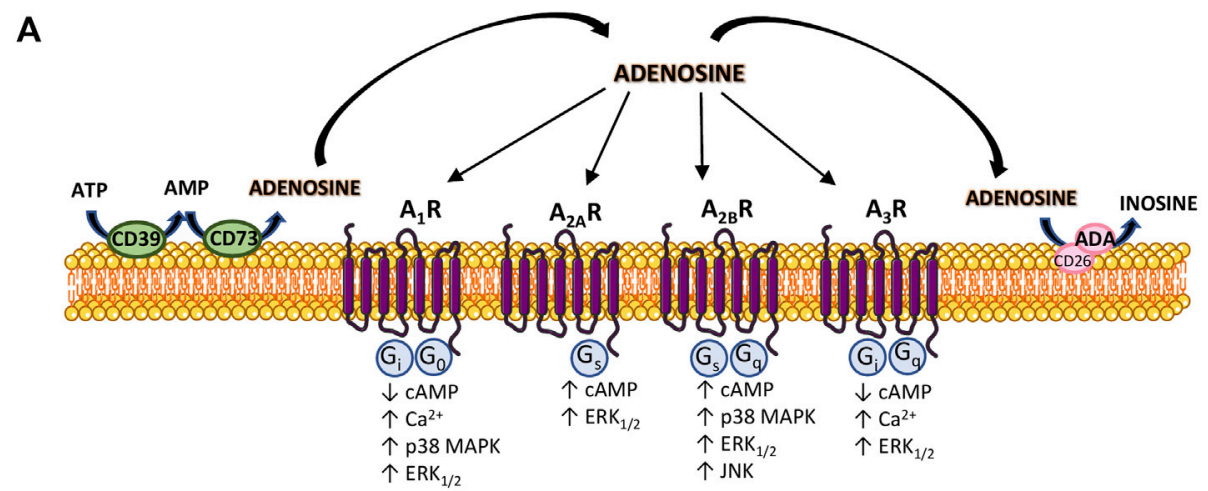

B

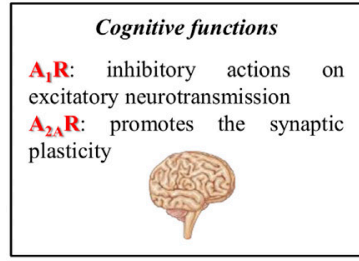

Metabolic homeostasis

$\mathbf{A}_{1} \mathbf{R}$ : inhibits lipolysis and $\uparrow$ insulin sensitivity

$\mathbf{A}_{2 \mathrm{~A}} \mathbf{R}$ : stimulates gluconeogenesis and glycogenolysis

$\mathbf{A}_{2 \mathrm{~B}} \mathbf{R}: \uparrow$ glucose and insulin

tolerance; $\uparrow$ lipid synthesis

$\mathbf{A}_{3} \mathbf{R}$ : stimulates gluconeogenesis

and glycogenolysis
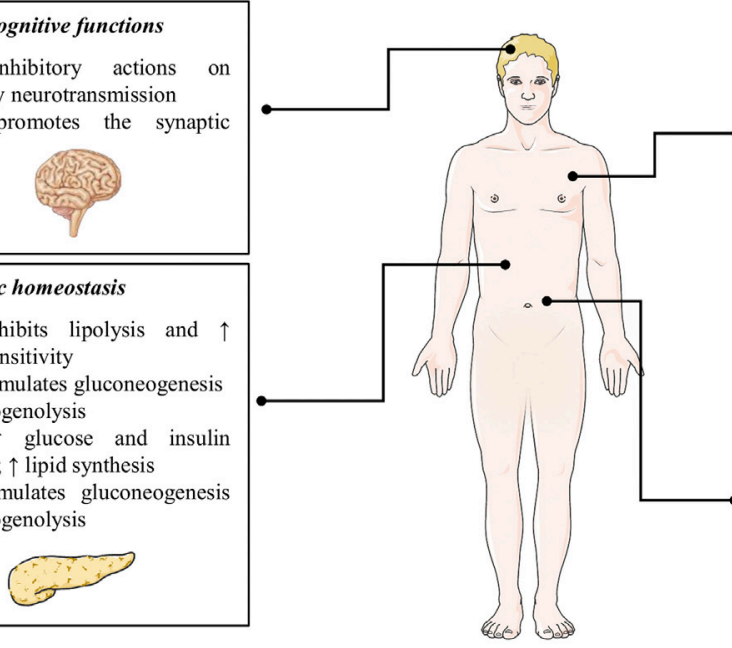

Cardiovascular system

$\mathbf{A}_{1} \mathbf{R}$ : negative chronotropic and dromotropic effects; $\downarrow \beta$ adrenergic tone; inhibition of $\mathrm{L}$ type calcium currents

$\mathbf{A}_{2,} \mathbf{R}$ : coronary and peripheral vasodilation; inhibition of platelet aggregation

$\mathbf{A}_{2 \mathrm{~B}} \mathbf{R}$ : vascular smooth muscle relaxation

$\mathbf{A}_{3} \mathbf{R}$ : vascular smooth muscle contraction

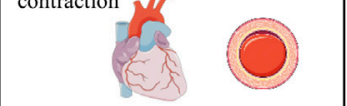

Gastrointestinal system

$\mathbf{A}_{1} \mathbf{R}$ : inhibitory actions on duodenal motility

$\mathbf{A}_{2 \mathbf{A}} \mathbf{R}$ : modulates bowel motor activity and enteric secretion

$\mathbf{A}_{2 \mathrm{~B}} \mathrm{R}$ : regulates intestinal secretion, motor function, and the immune response

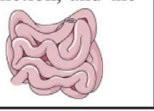

C

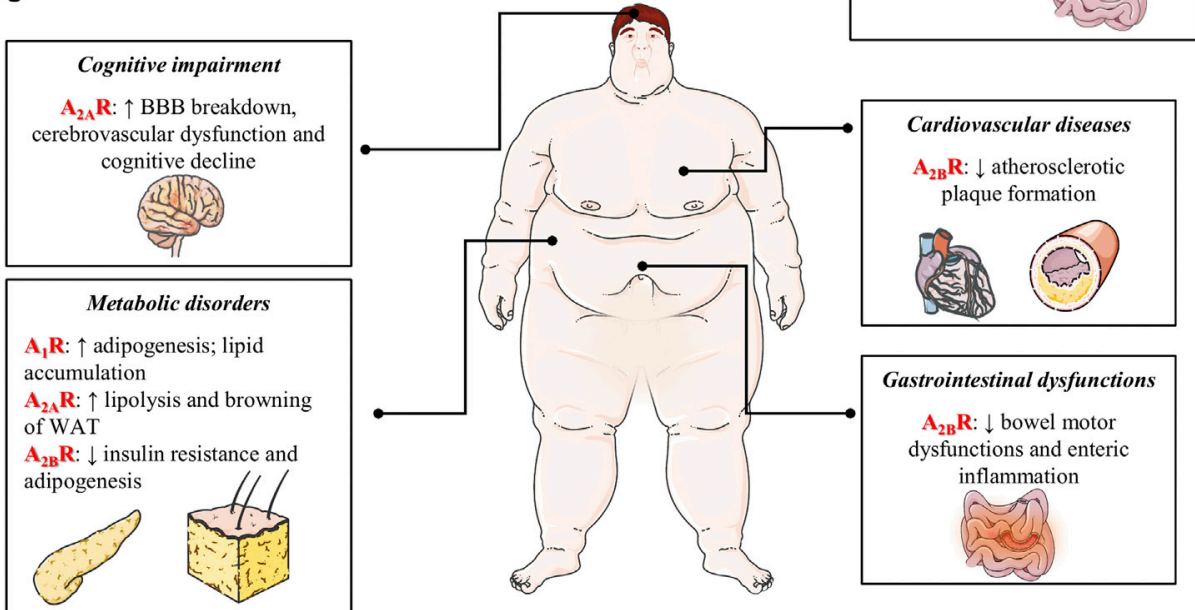

FIGURE 1 | (A) Adenosine receptor signaling. The extracellular adenosine is mainly produced by the ectonucleotidases, CD39 and CD73. Excess adenosine is irreversibly deaminated to inosine by $A D A$. Adenosine can bind to four different $G$ protein-coupled adenosine receptors, that either inhibit $\left(A_{1} R\right.$ and $\left.A_{3} R\right)$ or stimulate $\left(A_{2 A} R\right.$ and $\left.A_{2 B} R\right)$ adenylyl cyclase activity and cAMP production in the cell. All ARs are coupled also to MAPK pathways, including ERK $1 / 2$ and p38 MAPK. (B) Actions of adenosine receptors on various organ under physiological conditions. In $C N S, A_{1}$ Rs exert inhibitory actions on excitatory neurotransmission, while $A_{2 A}$ Rs promote excitatory actions. In cardiovascular system, the engagement of $A_{1} R$ s and $A_{3} R$ s triggers the vascular smooth muscle contraction, whereas the activation of $A_{2 A} R$ and $A_{2 B} R s$ mediates the vascular smooth muscle relaxation. In addition, $A_{1} R$ activation triggers negative chronotropic and dromotropic effects and $A_{2 A} R$ engagement inhibits platelet aggregation. In gastrointestinal system, the activation of $A_{1} R s, A_{2 A} R s$ and $A_{2 B} R$ regulates intestinal secretion and motor function. Furthermore, the activation of $A_{2 A} R s$ and $A_{3} R s$ stimulates gluconeogenesis and glycogenolysis; while, $A_{1} R s$ and $A_{2 B} R s$ inhibit lipolysis and increase the lipid synthesis. (C) Actions of adenosine 
FIGURE 1 | receptors on obesity and related comorbidities. The activation of $A_{1}$ Rs stimulates adipogenesis and lipid accumulation, thus promoting weight gain. By contrast, the activation of $A_{2 B} R s$ reduces adipogenesis, insulin resistance and atherosclerotic plaque formation; it counteracts also enteric inflammation and gastrointestinal dysfunction. The engagement of $\mathrm{A}_{2 \mathrm{~A}} \mathrm{Rs}$ triggers browning process and lipolysis, and mediates obesity-induced BBB breakdown and cognitive dysfunctions. $\uparrow$, increase; $\downarrow$, decrease. Abbreviations: $A_{1} R$, adenosine 1 receptor; $A_{2 A} R$, adenosine $2 A$ receptor; $A_{2 B} R$, adenosine $2 B$ receptor; $A_{3} R$, adenosine 3 receptor; $A D A$, adenosine deaminase; $A T P$, adenosine triphosphate; $B B B$, blood-brain barrier; $\mathrm{Ca}^{2+}$, calcium ions; $C A M P$, cyclic $A M P$; $C N S$, central nervous system; $E R K_{1 / 2}$, extracellular signal-regulated kinase 1/2; JNK, JUN N-terminal kinase; MAPK, mitogen-activated protein kinase; WAT, white adipose tissue.

also pivotal roles in modulating glucose homeostasis and insulin resistance, thus emerging as a promising target for drug development. Indeed, the systemic administration of the selective $A_{2 B} R$ agonist, BAY60-6583, reduced significantly the plasma glucose, insulin and IL-6 levels, and ameliorated T2DM in HFD mice (Johnston-Cox et al., 2014).

Overall, current data provide evidence that targeting $A_{1} R$ or $\mathrm{A}_{2 \mathrm{~B}} \mathrm{R}$ activation could represent a useful therapeutic strategy for preventing and/or treating obesity-related metabolic disorders.

\section{Cardiovascular Diseases}

Obesity is associated with an increased risk of developing CVDs, including hypertension, atherosclerosis and myocardial infarction (Carbone et al., 2019). Increasing evidence suggests that the excess of adipose tissue favors the secretion of proinflammatory cytokines and an overproduction of oxidant molecular species that affect the cardiovascular system directly or indirectly (Carbone et al., 2019).

Over the last years, the pharmacological modulation of ARs has emerged as a promising therapeutic approach also for the management of CVDs (Jacobson et al., 2019). In this regard, it has been reported that the activation of myocardial $A_{1}$ Rs by the partial agonist capadenoson protects from ischemia-reperfusion injury in a rat model of acute myocardial infarction, thus corroborating the evidence about a cardioprotective role of this receptor subtype (Albrecht-Kupper et al., 2012). Other authors observed an anti-hypertensive effect after treatment with the $\mathrm{A}_{2 \mathrm{~A}} \mathrm{R}$ agonist CGS21680 in a mouse model of hypertension induced by partial constriction of the renal artery, highlighting a modulatory role of $\mathrm{A}_{2 \mathrm{~A}} \mathrm{Rs}$ in regulating vascular smooth muscle relaxation/contraction (Schindler et al., 2005). Despite these interesting findings, currently only one preclinical study has investigated the effects of AR ligands on CVDs associated with obesity. In this setting, Koupenova et al. reported a beneficial effect of the $\mathrm{A}_{2 \mathrm{~B}} \mathrm{R}$ agonist BAY60-6583 on atherosclerotic plaque formation in HFD mice as a consequence of a decrease in cholesterol and triglyceride plasma levels (Koupenova et al., 2012). Despite the need for additional studies, this preliminary evidence suggests the pharmacological modulation of $\mathrm{A}_{2 \mathrm{~B}} \mathrm{R}$ as an intriguing strategy for the therapeutic management of CVDs associated with obesity.

\section{Gastrointestinal Dysfunctions}

Obesity is characterized by GI disturbances, including gastroesophageal reflux, irritable bowel syndrome, diarrhea and constipation (Camilleri et al., 2017; D'Antongiovanni et al., 2020a). Preclinical studies have shown that HFD animals, beyond showing alterations of gut microbiota and intestinal epithelial barrier, present a low-grade enteric inflammation, which contributes to the onset of intestinal dysfunctions associated with obesity (Bhattarai et al., 2016; Antonioli et al., 2017; Antonioli et al., 2020).

Increasing evidence highlights a critical role for the adenosine system in the pathophysiology of bowel dysfunctions associated with obesity. In particular, Antonioli et al. observed that adenosine, via $\mathrm{A}_{2 \mathrm{~B}} \mathrm{Rs}$, participates to obesity-related enteric dysmotility, modulating the activity of excitatory tachykininergic nerves in HFD mice (Antonioli et al., 2017). In support of this finding, a recent study confirmed the contribution of $\mathrm{A}_{2 \mathrm{~B}} \mathrm{Rs}$, expressed on enteric glial cells, in the modulation of tachykininergic responses and enteric inflammation associated with obesity, thus corroborating the relevant contribution of $\mathrm{A}_{2 \mathrm{~B}} \mathrm{Rs}$ to the pathophysiology of bowel motor dysfunctions and inflammation associated with obesity (D'Antongiovanni et al., 2020c). Based on these data, the pharmacological modulation of $\mathrm{A}_{2 \mathrm{~B}} \mathrm{R}$ represents a viable way to design novel tools to manage both bowel motor dysfunctions and enteric inflammation associated with obesity.

\section{Cognitive Impairment}

Increasing evidence support the concept that obesity can lead to neuroinflammation, and neurodegenerative diseases (i.e., Alzheimer's disease), and can affect negatively cognition, including attention and decision making (Leigh and Morris, 2020). In line with this view, obese mice display brain dysfunctions and learning impairment (Leigh and Morris, 2020).

Adenosine has been shown to play a critical role in the control of the cognitive functions (Choudhury et al., 2019). In particular, adenosine was found to exert both inhibitory actions on excitatory neurotransmission, via $\mathrm{A}_{1} \mathrm{R}$ activation, and excitatory actions driven by engagement of $\mathrm{A}_{2 \mathrm{~A}}$ Rs (Gomes et al., 2011). In the context of obesity, a limited number of experimental studies have been focused on the possible involvement of ARs in the pathophysiology of cognitive impairment. For instance, Yamamoto et al. investigated the role of $\mathrm{A}_{2 \mathrm{~A}} \mathrm{Rs}$ in obesity-induced cognitive dysfunctions. They described an increase in blood-brain barrier (BBB) breakdown along with cognitive impairment in HFD mice, and observed that the genetic ablation of $\mathrm{A}_{2 \mathrm{~A}} \mathrm{Rs}$ in endothelial cells protected HFD mice against the $\mathrm{BBB}$ impairment and cognitive dysfunction (Yamamoto et al., 2019). This study provides the first evidence that changes in cerebrovascular permeability initiate the cycle of obesity-induced neuroinflammation and cognitive impairment. In this context, the pharmacological modulation of $\mathrm{A}_{2 \mathrm{~A}} \mathrm{Rs}$ could be considered as an interesting molecular target to design novel therapeutic strategies aimed at managing the cognitive impairment associated with obesity. 


\section{CONCLUSION}

Nowadays, the therapeutic management of obese patients remains unsatisfactory. A body of preclinical knowledge indicates that the pharmacological modulation of adenosine system has a promising potential for treating obesity and related comorbidities. In this regard, the development of experimental models of obesity, including diet-induced models as well as the genetic models (i.e. leptin-deficient model) allowed to better understand the role of adenosine system in the pathophysiological mechanisms underlying obesity. Indeed, adenosine modulates actively glucose homeostasis, inflammation, adipogenesis, insulin resistance and thermogenesis, depending on the engagement of receptor subtypes in different tissues (Fredholm, 2014). For instance, $\mathrm{A}_{1} \mathrm{R}$ activation stimulates adipogenesis and lipid accumulation, thus promoting weight gain. Accordingly, future efforts should be focused on investigating better the effects of $A_{1} R$ ligands on lipogenic and thermogenic processes in the context of obesity. The activation of $\mathrm{A}_{2 \mathrm{~A}} \mathrm{Rs}$ can trigger the browning process of WAT, support the lipolytic process, and counteract significantly the obesity-induced $\mathrm{BBB}$ alterations and cognitive dysfunctions. Along the same line, the pharmacological stimulation of $\mathrm{A}_{2 \mathrm{~B}} \mathrm{Rs}$ can exert beneficial effects on obesity, since these receptors participate actively in shaping adipogenesis, insulin resistance, inflammation, atherosclerotic plaque formation and GI dysfunctions associated with obesity (Figure 1C). However, the paucity of selective $\mathrm{A}_{2 \mathrm{~B}} \mathrm{R}$ ligands limits greatly the exploration of the therapeutic implications of these receptor subtypes. Therefore, the synthesis of highly selective compounds is needed, particularly to curb the occurrence of

\section{REFERENCES}

Albrecht-Küpper, B. E., Leineweber, K., and Nell, P. G. (2012). Partial adenosine A1 receptor agonists for cardiovascular therapies. Purinergic Signal. 8, 91-99. doi:10.1007/s11302-011-9274-3

Antonioli, L., Colucci, R., La Motta, C., Tuccori, M., Awwad, O., Da Settimo, F., et al. (2012). Adenosine deaminase in the modulation of immune system and its potential as a novel target for treatment of inflammatory disorders. Curr. Drug Targets. 13, 842-862. doi:10.2174/138945012800564095

Antonioli, L., D’Antongiovanni, V., Pellegrini, C., Fornai, M., Benvenuti, L., Di Carlo, A., et al. (2020). Colonic dysmotility associated with high-fat dietinduced obesity: role of enteric glia. FASEB J. 34, 5512-5524. doi:10.1096/fj. 201901844R

Antonioli, L., Fornai, M., Colucci, R., Awwad, O., Ghisu, N., Tuccori, M., et al. (2011). Differential recruitment of high affinity A1 and A2A adenosine receptors in the control of colonic neuromuscular function in experimental colitis. Eur. J. Pharmacol. 650, 639-649. doi:10.1016/j.ejphar. 2010.10 .041

Antonioli, L., Pacher, P., Vizi, E. S., and Haskó, G. (2013). CD39 and CD73 in immunity and inflammation. Trends Mol. Med. 19, 355-367. doi:10.1016/j. molmed.2013.03.005

Antonioli, L., Pellegrini, C., Fornai, M., Tirotta, E., Gentile, D., Benvenuti, L., et al. (2017). Colonic motor dysfunctions in a mouse model of high-fat diet-induced obesity: an involvement of A2B adenosine receptors. Purinergic Signal. 13, 497-510. doi:10.1007/s11302-017-9577-0

Bhattarai, Y., Fried, D., Gulbransen, B., Kadrofske, M., Fernandes, R., Xu, H., et al. (2016). High-fat diet-induced obesity alters nitric oxide-mediated neuromuscular transmission and smooth muscle excitability in the mouse adverse effects. At present, no data are available about the involvement of $\mathrm{A}_{3}$ Rs in the onset and development of obesityassociated comorbidities.

Despite current evidences arising from the animal models are encouraging, the translation of preclinical data into clinical practice will require a more thorough understanding of the tissue-specific effects of adenosine. At present, the main point of weakness concerns the limited methods to quantify the adenosine and $\mathrm{ADA}$ activity in vivo.

Over the years, a growing body of preclinical evidence has highlighted the possibility of beneficial effects resulting from the pharmacological modulation of adenosine pathways in the context of obesity. However, a number of issues regarding the regulatory role of digestive functions by the adenosine system are still pending and deserve further investigations. Of note, the available clinical data regarding the involvement of the adenosine system in obesity are scanty, and it is not possible to have consolidated evidence or draw substantial conclusions.

Taken together, the pharmacological modulation of the adenosine system represents an attractive strategy for the scientific community, encouraging the development of novel AR ligands useful to manage and counteract obesity and its related comorbidities.

\section{AUTHOR CONTRIBUTIONS}

VD and LA literature search, writing original draft preparation; $\mathrm{CP}$ helped in the preparation of the figures, and reviewed the draft; $M F$ and $C B$ reviewed the draft. All authors have read and agreed to the published version of the manuscript.

distal colon. Am. J. Physiol. Gastrointest. Liver Physiol. 311, G210-G220. doi:10. 1152/ajpgi.00085.2016

Blüher, M. (2019). Obesity: global epidemiology and pathogenesis. Nat. Rev. Endocrinol. 15, 288-298. doi:10.1038/s41574-019-0176-8

Boison, D. (2013). Adenosine kinase: exploitation for therapeutic gain. Pharmacol. Rev. 65, 906-943. doi:10.1124/pr.112.006361

Burnstock, G., and Gentile, D. (2018). The involvement of purinergic signalling in obesity. Purinergic Signal. 14, 97-108. doi:10.1007/s11302-018-9605-8

Camilleri, M., Malhi, H., and Acosta, A. (2017). Gastrointestinal complications of obesity. Gastroenterology. 152, 1656-1670. doi:10.1053/j.gastro.2016.12.052

Carbone, S., Canada, J. M., Billingsley, H. E., Siddiqui, M. S., Elagizi, A., and Lavie, C. J. (2019). Obesity paradox in cardiovascular disease: where do we stand? Vasc. Health Risk Manag. 15, 89-100. doi:10.2147/VHRM.S168946

Choudhury, H., Chellappan, D. K., Sengupta, P., Pandey, M., and Gorain, B. (2019). Adenosine receptors in modulation of central nervous system disorders. Curr. Pharmaceut. Des. 25, 2808-2827. doi:10.2174/1381612825666190712181955

Csóka, B., Koscsó, B., Töro, G., Kókai, E., Virág, L., Németh, Z. H., et al. (2014). $\mathrm{A} 2 \mathrm{~B}$ adenosine receptors prevent insulin resistance by inhibiting adipose tissue inflammation via maintaining alternative macrophage activation. Diabetes. 63, 850-866. doi:10.2337/db13-0573

Cully, M. (2014). Obesity: adenosine protects from diet-induced obesity. Nat. Rev. Drug Discov. 13, 886-887. doi:10.1038/nrd4490

D’Antongiovanni, V., Pellegrini, C., Fornai, M., Colucci, R., Blandizzi, C., Antonioli, L., et al. (2020a). Intestinal epithelial barrier and neuromuscular compartment in health and disease. World J. Gastroenterol. 26, 1564-1579. doi:10.3748/wjg.v26.i14.1564

D’Antongiovanni, V., Fornai, M., Pellegrini, C., Benvenuti, L., Blandizzi, C., and Antonioli, L. (2020b). The adenosine system at the crossroads of intestinal inflammation and Neoplasia. Int. J. Mol. Sci. 21, 54. 
D’Antongiovanni, V., Benvenuti, L., Fornai, M., Pellegrini, C., Van Den Wijngaard, R., Cerantola, S., et al. (2020c). Glial A2B adenosine receptors modulate abnormal tachykininergic responses and prevent enteric inflammation associated with high fat diet-induced obesity. Cells. 9, 21.

Da Silva, C. G., Jarzyna, R., Specht, A., and Kaczmarek, E. (2006). Extracellular nucleotides and adenosine independently activate AMP-activated protein kinase in endothelial cells: involvement of P2 receptors and adenosine transporters. Circ. Res. 98, e39-47. doi:10.1161/01.RES.0000215436.92414.1d

Dong, Q., Ginsberg, H. N., and Erlanger, B. F. (2001). Overexpression of the A1 adenosine receptor in adipose tissue protects mice from obesity-related insulin resistance. Diabetes Obes. Metabol. 3, 360-366. doi:10.1046/j.1463-1326.2001. 00158.x

Eisenstein, A., Carroll, S. H., Johnston-Cox, H., Farb, M., Gokce, N., and Ravid, K. (2014). An adenosine receptor-Krüppel-like factor 4 protein axis inhibits adipogenesis. J. Biol. Chem. 289, 21071-21081. doi:10.1074/jbc.M114.566406

Ellulu, M. S., Patimah, I., Khaza'ai, H., Rahmat, A., and Abed, Y. (2017). Obesity and inflammation: the linking mechanism and the complications. Arch. Med. Sci. 13, 851-863. doi:10.5114/aoms.2016.58928

Eltzschig, H. K. (2013). Extracellular adenosine signaling in molecular medicine. J. Mol. Med. (Berl.). 91, 141-146. doi:10.1007/s00109-013-0999-z

Figler, R. A., Wang, G., Srinivasan, S., Jung, D. Y., Zhang, Z., Pankow, J. S., et al. (2011). Links between insulin resistance, adenosine A2B receptors, and inflammatory markers in mice and humans. Diabetes. 60, 669-679. doi:10. 2337/db10-1070

Fredholm, B. B. (2014). Adenosine-a physiological or pathophysiological agent?. J. Mol. Med. (Berl.). 92, 201-206. doi:10.1007/s00109-013-1101-6

Gharibi, B., Abraham, A. A., Ham, J., and Evans, B. A. (2012). Contrasting effects of $\mathrm{A} 1$ and A2b adenosine receptors on adipogenesis. Int. J. Obes. 36, 397-406. doi:10.1038/ijo.2011.129

Gnad, T., Scheibler, S., von Kügelgen, I., Scheele, C., Kilić, A., Glöde, A., et al. (2014). Adenosine activates brown adipose tissue and recruits beige adipocytes via A2A receptors. Nature. 516, 395-399. doi:10.1038/nature13816

Gomes, C. V., Kaster, M. P., Tomé, A. R., Agostinho, P. M., and Cunha, R. A. (2011). Adenosine receptors and brain diseases: neuroprotection and neurodegeneration. Biochim. Biophys. Acta. 1808, 1380-1399. doi:10.1016/j. bbamem.2010.12.001

Hardy, O. T., Czech, M. P., and Corvera, S. (2012). What causes the insulin resistance underlying obesity?. Curr. Opin. Endocrinol. Diabetes Obes. 19, 81-87. doi:10.1097/MED.0b013e3283514e13

Haskó, G., Linden, J., Cronstein, B., and Pacher, P. (2008). Adenosine receptors: therapeutic aspects for inflammatory and immune diseases. Nat. Rev. Drug Discov. 7, 759-770. doi:10.1038/nrd2638

Jacobson, K. A., Tosh, D. K., Jain, S., and Gao, Z. G. (2019). Historical and current adenosine receptor agonists in preclinical and clinical development. Front. Cell. Neurosci. 13, 124. doi:10.3389/fncel.2019.00124

Jarolimova, J., Tagoni, J., and Stern, T. A. (2013). Obesity: its epidemiology, comorbidities, and management. Prim. Care Companion CNS Disord. 15, 64. doi:10.4088/PCC.12f01475

Johnston-Cox, H., Eisenstein, A. S., Koupenova, M., Carroll, S., and Ravid, K. (2014). The macrophage A2B adenosine receptor regulates tissue insulin sensitivity. PLoS One. 9, e98775. doi:10.1371/journal.pone.0098775
Kotańska, M., Dziubina, A., Szafarz, M., Mika, K., Reguła, K., Bednarski, M., et al (2020). KD-64-A new selective A2A adenosine receptor antagonist has antiinflammatory activity but contrary to the non-selective antagonist-Caffeine does not reduce diet-induced obesity in mice. PloS One. 15, e0229806. doi:10. 1371/journal.pone.0229806

Koupenova, M., Johnston-Cox, H., Vezeridis, A., Gavras, H., Yang, D., Zannis, V. et al. (2012). A2b adenosine receptor regulates hyperlipidemia and atherosclerosis. Circulation. 125, 354-363. doi:10.1161/ CIRCULATIONAHA.111.057596

Leigh, S. J., and Morris, M. J. (2020). Diet, inflammation and the gut microbiome: mechanisms for obesity-associated cognitive impairment. Biochim. Biophys Acta (BBA) - Mol. Basis Dis. 1866, 165767. doi:10.1016/j.bbadis.2020.165767

Mohell, N. (1984). Alpha 1-adrenergic receptors in brown adipose tissue. Thermogenic significance and mode of action. Acta Physiol. Scand. Suppl., $530,1-62$.

Nowotny, K., Jung, T., Höhn, A., Weber, D., and Grune, T. (2015). Advanced glycation end products and oxidative stress in type 2 diabetes mellitus. Biomolecules. 5, 194-222. doi:10.3390/biom5010194

Ouchi, N., Parker, J. L., Lugus, J. J., and Walsh, K. (2011). Adipokines in inflammation and metabolic disease. Nat. Rev. Immunol. 11, 85-97. doi:10. 1038/nri2921

Pardo, F., Villalobos-Labra, R., Chiarello, D. I., Salsoso, R., Toledo, F., Gutierrez, J., et al. (2017). Molecular implications of adenosine in obesity. Mol. Aspect. Med. 55, 90-101. doi:10.1016/j.mam.2017.01.003

Pastor-Anglada, M., Urtasun, N., and Pérez-Torras, S. (2018). Intestinal nucleoside transporters: function, expression, and regulation. Comp. Physiol. 8, 1003-1017. doi:10.1002/cphy.c170039

Schindler, C. W., Karcz-Kubicha, M., Thorndike, E. B., Müller, C. E., Tella, S. R., Ferré, S., et al. (2005). Role of central and peripheral adenosine receptors in the cardiovascular responses to intraperitoneal injections of adenosine A1 and A2A subtype receptor agonists. Br. J. Pharmacol. 144, 642-650. doi:10.1038/sj.bjp. 0706043

Sheth, S., Brito, R., Mukherjea, D., Rybak, L. P., and Ramkumar, V. (2014). Adenosine receptors: expression, function and regulation. Int. J. Mol. Sci. 15, 2024-2052. doi:10.3390/ijms15022024

Yamamoto, M., Guo, D. H., Hernandez, C. M., and Stranahan, A. M. (2019). Endothelial Adora2a activation promotes blood-brain barrier breakdown and cognitive impairment in mice with diet-induced insulin resistance. J. Neurosci. 39, 4179-4192. doi:10.1523/JNEUROSCI.2506-18.2019

Conflict of Interest: The authors declare that the research was conducted in the absence of any commercial or financial relationships that could be construed as a potential conflict of interest.

Copyright (C) 2021 D'Antongiovanni, Fornai, Pellegrini, Blandizzi and Antonioli. This is an open-access article distributed under the terms of the Creative Commons Attribution License (CC BY). The use, distribution or reproduction in other forums is permitted, provided the original author(s) and the copyright owner(s) are credited and that the original publication in this journal is cited, in accordance with accepted academic practice. No use, distribution or reproduction is permitted which does not comply with these terms. 